\section{Depuis sa création jusqu'à}

son altération, la vie d'une œuvre

\section{d'art ou d'un objet archéologique}

s'inscrit au cœur de sa matière.

L'enjeu des sciences du patrimoine

\section{est d'interroger cette matière}

sur l'activité des hommes,

tout en préservant ces témoins

de notre histoire. Retrouver

les savoir-faire du passé,

comprendre le choix des matériaux

employés et déterminer l'origine

des matières premières en sont

les principales motivations.

En révélant directement et sans

dommages la composition

élémentaire des matériaux

anciens, l'analyse par faisceau

d'ions réalisée à AGLAÉ

(Accélérateur Grand Louvre

pour l'Analyse Élémentaire)

est parfaitement adaptée

aux problématiques des sciences

du patrimoine.

\title{
Un accélérateur de particules fait parler les œuvres d'art et les objets archéologiques
}

Thomas Calligaro (thomas.calligaro@culture.fr) et Claire Pacheco

Centre de recherche et de restauration des musées de France (C2RMF)

Palais du Louvre, 14 Quai François Mitterand, 75001 Paris

\section{Analyse par faisceau d'ions}

Regroupées sous le sigle IBA pour Ion Beam Analysis, les méthodes d'analyse par faisceaux d'ions sont une retombée inattendue de la physique nucléaire. Elles résultent de la conjonction, dans les années 1970, de trois opportunités : l'accès aux catalogues des niveaux d'énergie et des réactions des noyaux, la modélisation détaillée de l'interaction rayonnement-matière et l'invention des détecteurs à semi-conducteurs.

Les méthodes d'analyse IBA exploitent les radiations (rayons $\mathrm{X}$, rayons $\gamma$ et particules chargées) émises par une cible inconnue sous l'impact du faisceau de particules produit par un accélérateur, pour déterminer sa composition. L'énergie et le type des radiations émises permettent d'identifier les éléments présents et de les quantifier. Les particules incidentes sont des ions légers (par exemple protons ${ }^{1} \mathrm{H}^{+}$, noyaux de deutérium ${ }^{2} \mathrm{H}^{+}$ou deutons, noyaux d'hélium ${ }^{4} \mathrm{He}^{2+}$ ) accélérés à quelques $\mathrm{MeV}$ (millions d'électronvolts), énergie optimale pour l'analyse des matériaux. La vitesse de ces ions est comparable à celle des électrons de cœur des atomes de la cible, maximisant le processus d'ionisation, et leur permet de surmonter la répulsion coulombienne des noyaux des atomes les plus légers pour induire une réaction nucléaire.
La perte d'énergie linéique de ces ions est minimale et leur parcours dans la matière atteint plusieurs dizaines de microns $(\mu \mathrm{m})$ (par exemple les protons de $1 \mathrm{MeV}$ pénètrent à $40 \mu \mathrm{m}$ dans un bronze et à $100 \mu \mathrm{m}$ dans un verre).

L'ion incident peut interagir avec la cible selon trois processus différents, schématisés sur la figure 1, et qui sont à la base des méthodes IBA [1].

- Éjection d'un électron de cœur d'un atome de la cible, provoquant l'émission de rayons $\mathrm{X}$ caractéristiques. Analogue à l'analyse par fluorescence des rayons X, cette méthode, nommée PIXE pour Particle-Induced X-ray Emission, permet de quantifier les éléments à partir du sodium avec une sensibilité qui peut atteindre quelques parties par million (ppm). Les particules incidentes sont généralement des protons de $3 \mathrm{MeV}$.

- Rétrodiffusion élastique par répulsion électrostatique d'un noyau de la cible. L'énergie des ions rétrodiffusés augmente avec la masse du noyau rétrodiffusant. Cette méthode, nommée RBS pour Rutherford Backscattering Spectrometry, permet d'identifier les éléments par leur masse et de les localiser en profondeur. Elle est très efficace pour caractériser des couches d'éléments lourds déposés sur un substrat d'éléments légers. Les particules incidentes optimales sont des ions ${ }^{4} \mathrm{He}$ de $3 \mathrm{MeV}$. 


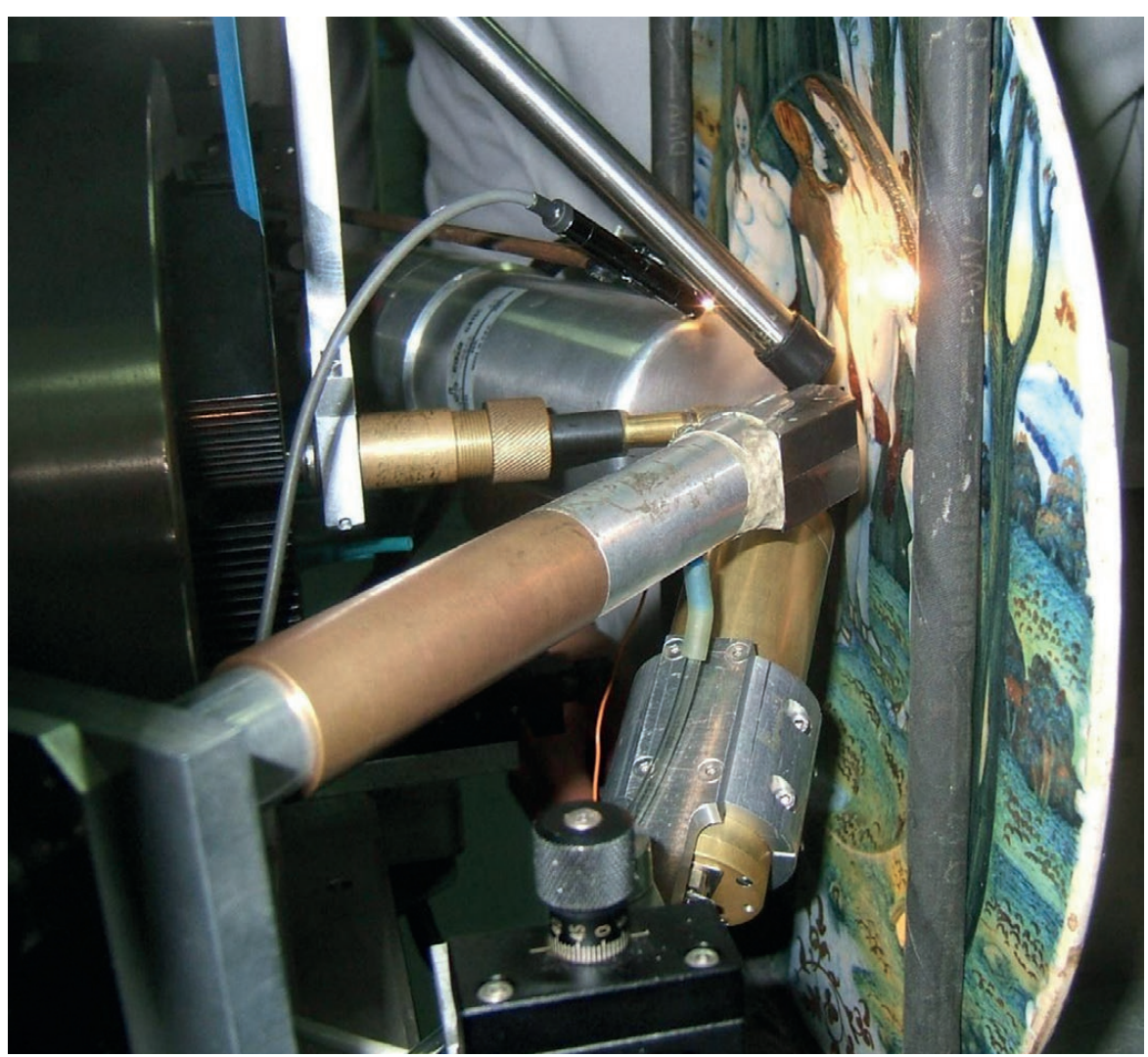

Plat à décor lustré (Jugement de Pâris, 1520, Maestro Gorgio, Gubbio, Italie) placé dans le faisceau extrait de l'accélérateur AGLAÉ.
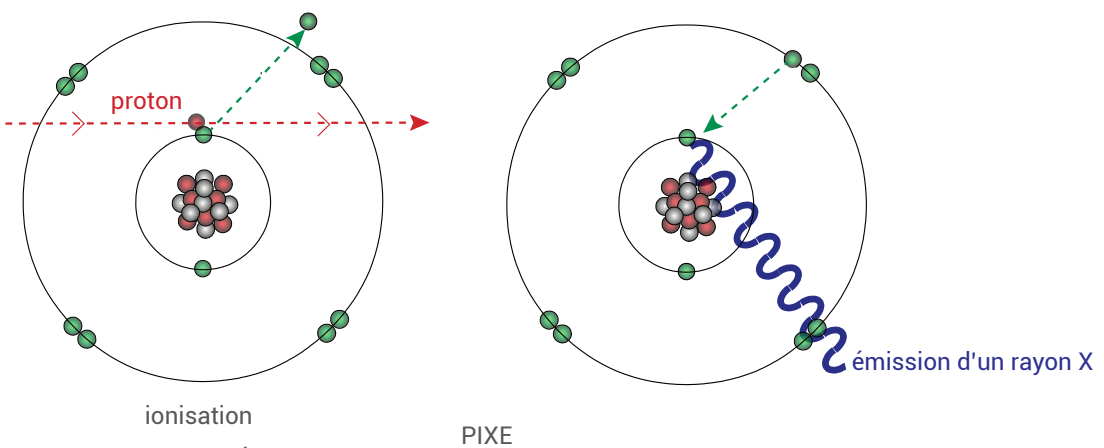

Émission $\mathrm{X}$ induite par particules chargées

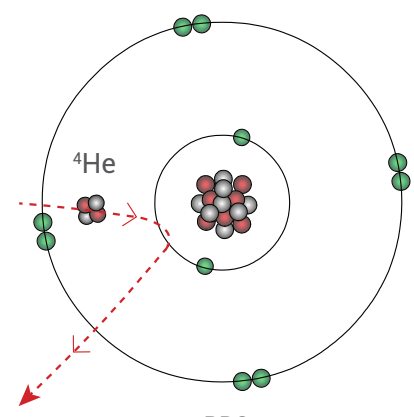

RBS
Spectroscopie de rétrodiffusion Rutherford

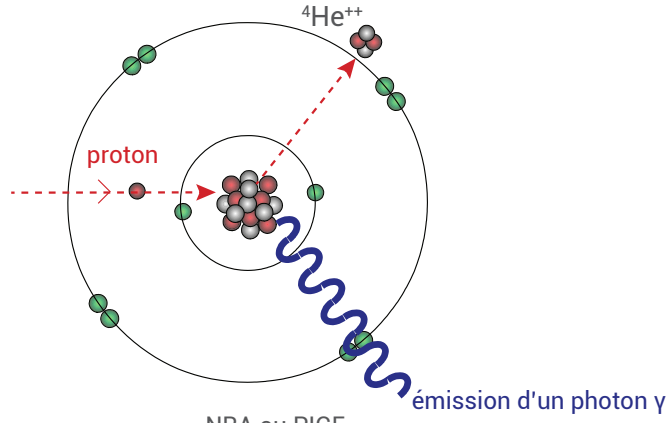

NRA ou PIGE

Analyse par réaction nucléaire
1. Schémas de principe des trois grandes classes de méthode d'analyse par faisceau d'ions. En haut, la méthode PIXE procède en deux étapes : expulsion d'un électron de cœur, puis réarrangement électronique accompagné de l'émission d'un rayon $\mathrm{X}$ caractéristique.

En bas, la rétrodiffusion de l'ion incident (RBS) par répulsion coulombienne du noyau d'un atome cible et une réaction nucléaire (NRA ou PIGE) entre la particule incidente et le noyau d'un atome de la cible.
- Interaction nucléaire avec le noyau d'un atome de la cible. Un noyau composé, formé dans un état excité, se désintègre ou se désexcite en émettant une radiation : ion incident + noyau cible $\rightarrow$ noyau composé $\rightarrow$ noyau final + radiation. Cette méthode se nomme NRA pour Nuclear Reaction Analysis lorsque la radiation émise est une particule chargée, PIGE pour Particle Induced Gamma-ray Emission lorsque c'est un photon $\gamma$. Les méthodes NRA et PIGE permettent de quantifier un isotope particulier; elles sont l'analogue pour les éléments légers des méthodes RBS et PIXE. Les ions incidents sont le plus souvent des protons ou des deutons de quelques $\mathrm{MeV}$.

Les méthodes IBA exploitent le comportement opposé des photons et des ions traversant la matière. Les photons conservent leur énergie mais leur nombre décroit par absorption, alors que les ions restent en nombre constant et leur énergie diminue jusqu'à l'arrêt. L'énergie des rayons $\mathrm{X}$ et $\gamma$ ne dépend donc pas de la profondeur à laquelle ils ont été émis, d'où une faible résolution en profondeur des méthodes PIXE et PIGE. À l'inverse, 
$>>$

les méthodes RBS et NRA peuvent localiser en profondeur les éléments, car l'énergie des ions dépend de l'épaisseur traversée. La profondeur analysée varie entre quelques microns et des dizaines de microns selon la méthode IBA employée et l'élément mesuré. Dans le cas de la méthode PIXE, elle est conditionnée par l'énergie des raies caractéristiques des atomes. Pour les éléments légers, elle est limitée par l'absorption de leurs rayons X caractéristiques de faible énergie dans l'échantillon (par exemple $3 \mu \mathrm{m}$ pour le sodium dans un verre). Lorsque les rayons $\mathrm{X}$ caractéristiques sont de plus haute énergie, ou si ce sont des rayons $\gamma$ (PIGE), la profondeur sondée peut s'étendre jusqu'à la fin du parcours des ions incidents.

La mise en œuvre de ces méthodes est illustrée par deux études (pp. 18-19) menées avec l'accélérateur AGLAÉ installé au Palais du Louvre (voir l'encadré ci-dessous).

\section{Grandes questions en sciences du patrimoine}

La caractérisation des matériaux du patrimoine (composition, structure et disposition des éléments) permet de révéler les constituants et les techniques de manufacture du passé (par exemple recettes d'élaboration des verres), ou d'identifier les choix et techniques de l'artiste (par exemple palette de pigments). Les éléments présents à l'état de traces sont de précieux indicateurs de l'origine des matériaux employés. Ils nous renseignent sur l'approvisionnement en matières premières (par exemple provenance des minéraux) et sur les réseaux d'échange d'objets finis (par exemple circulation des céramiques). Enfin, l'environnement impacte nombre de matériaux et influe sur l'état de conservation des œuvres (par exemple corrosion des métaux). L'analyse de la surface des objets permet de traquer les signes d'altération, de comprendre les processus de dégradation pour stopper leur progression, de recommander des conditions de conservation plus adaptées et de guider les restaurations.

\section{Particularités des objets du patrimoine}

Les objets du patrimoine présentent des particularités et des contraintes spécifiques. Leur surface est rarement lisse et leur composition souvent hétérogène : en grains (céramiques), en couches (décors) ou composite organique/inorganique (tableaux). Cette complexité multi-échelle requiert des techniques analytiques possédant une bonne résolution latérale et en profondeur. Le caractère précieux et souvent unique des œuvres impose des méthodes qui évitent tout prélèvement ou modification. Leurs grandes dimensions et formes compliquées (par exemple sculptures) imposent une analyse directe à l'atmosphère.

L'accélérateur d'ions AGLAÉ en quelques mots

L'Accélérateur Grand Louvre pour l'Analyse Élementaire (AGLAÉ) est un tandem électrostatique de $2 \mathrm{MV}$ installé en 1989 dans les locaux du C2RMF au Palais du Louvre.

Les ions négatifs $\left(\mathrm{H}^{-}, \mathrm{He}^{-} \ldots\right)$ produits par les sources sont attirés par le potentiel positif du terminal, dans lequel ils sont « déshabillés " de leurs électrons au passage dans un gaz, le stripper, ce qui génère des ions chargés positivement $\left(\mathrm{H}^{+}, \mathrm{He}^{2+} \ldots\right)$ qui sont alors repoussés vers la sortie de l'accélérateur. Le faisceau de particules ainsi accéléré à une vitesse de l'ordre de $10 \%$ de la vitesse de la lumière, est focalisé et extrait à l'air en traversant une fine membrane de $\mathrm{Si}_{3} \mathrm{~N}_{4}$ de $100 \mathrm{~nm}$ d'épaisseur.

Les performances d'AGLAÉ ont été améliorées continuellement depuis son ouverture, mais certaines limites sont apparues au bout de vingt ans de services remarquables rendus aux chercheurs et aux conservateurs de musées : - absence d'automatisation de l'appareil et manque de stabilité du faisceau qui limitaient son temps d'utilisation à la journée et ne permettaient pas l'analyse en toute sécurité d'œuvres fragiles, comme les tableaux par exemple, qui peuvent présenter des dommages sous le faisceau en raison de la présence de matière organique ;

- difficulté de réaliser des cartographies chimiques systématiques ;

- utilisation difficile d'un faisceau de noyaux de deutérium $\left({ }^{2} \mathrm{H}^{+}\right)$qui permet d'enrichir la panoplie d'analyses par réactions nucléaires, et rend nécessaire le renforcement des conditions de radioprotection.

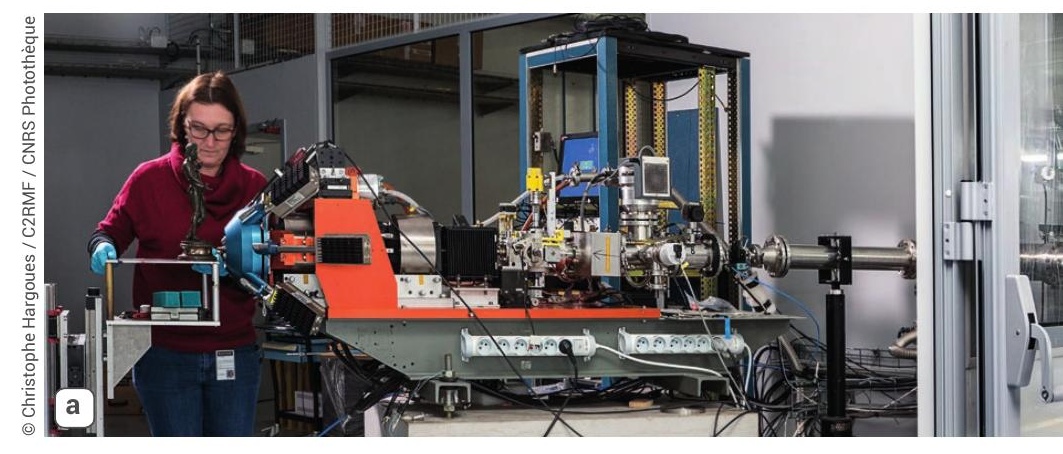

Financé à partir de 2012 par le programme d'investissements d'avenir de l'Agence nationale de la recherche (ANR-10EQPX-22), le ministère de la Culture, le CNRS et la Mairie de Paris (projet New AGLAÉ), le nouvel accélérateur AGLAÉ (fig. E1) a été inauguré en novembre 2017.

L'importante tâche de stabilisation du faisceau et l'automatisation de la ligne ont été réalisées de 2016 à 2017 en collaboration avec la société Thalès.

Concernant l'imagerie systématique, une surface maximale de $200 \times 200 \mathrm{~mm}^{2}$ est parcourue en combinant un balayage vertical du faisceau au moyen de bobines électromagnétiques et le déplacement mécanique de l'objet. Le mode d'acquisition sélectionné pour le nouvel AGLAÉ permet de corréler chaque rayonnement détecté avec la position $(X, Y)$ du faisceau sur la cible. Ce couplage permet de reconstruire la carte de répartition spatiale des données IBA [7]. 
la réaction ${ }^{2} \mathrm{H}+{ }^{12} \mathrm{C} \rightarrow{ }^{13} \mathrm{C}+{ }^{1} \mathrm{H}+\gamma$ permet, par le comptage des rayons $\gamma$ de 3,09 MeV (PIGE), de déterminer la concentration globale en carbone, et par celui des protons émis (NRA) de déterminer son profil en profondeur. L'hydrogène, élément léger impliqué dans les phénomènes d'hydratation peut être mesuré par la réaction nucléaire induite par des ions incidents ${ }^{15} \mathrm{~N}$, avec émission d'un rayon $\gamma$ de 4,44 $\mathrm{MeV}$ $\left({ }^{15} \mathrm{~N}+{ }^{1} \mathrm{H} \rightarrow{ }^{12} \mathrm{C}+{ }^{4} \mathrm{He}+\gamma\right)$. Cette réaction, dite résonante, se produit précisément lorsque les ions ${ }^{15} \mathrm{~N}$ ont une énergie de 6,385 MeV. En augmentant progressivement l'énergie des ions ${ }^{15} \mathrm{~N}$, l'hydrogène est sondé en profondeur et son profil de concentration peut être reconstitué. À ces méthodes IBA peuvent s'en adjoindre d'autres, comme la ionoluminescence (émission de lumière sous le faisceau) qui délivre des informations complémentaires à l'analyse élémentaire, notamment la présence de défauts.

Les capacités d'imagerie élémentaire à grande échelle des méthodes IBA sont remarquables. Le faisceau d'ions peut être focalisé sur moins d'un micron dans le vide ou sur une trentaine de microns dans l'atmosphère, et déplacé à la surface de la cible à l'aide de champs électriques et magnétiques. La combinaison du balayage rapide du faisceau à la surface de la cible avec un déplacement mécanique de l'objet permet de produire des cartes de répartition des éléments sur des dizaines de centimètres carrés avec une résolution latérale limitée par la taille du faisceau dans l'atmosphère, soit 50 microns. Le croisement de ces images avec l'imagerie optique est particulièrement informatif. Ces possibilités sont illustrées par le deuxième exemple sur l'imagerie PIXE/ RBS d'un vitrail.

Des outils quantitatifs de simulation et de traitement des spectres sont disponibles. Toutes les étapes de la physique des méthodes IBA sont formalisées et quantifiables. Les données fondamentales nécessaires au calcul (sections efficaces d'interaction, perte d'énergie des ions dans la matière, coefficients d'atténuation des rayons $X$ et $\gamma$ ) sont affinées régulièrement, et les valeurs obtenues sont versées dans des bases de données partagées par la communauté des utilisateurs de faisceaux d'ions. Des codes de calcul performants ont été développés [4], notamment les programmes GUPIXWIN (PIXE), SIMNRA (RBS/NRA/PIGE) et DATA
FURNACE (RBS et NRA) qui permettent d'obtenir les concentrations élémentaires et les profils en profondeur à partir des spectres IBA avec une précision de quelques pourcents. Leur principe consiste généralement à calculer le spectre produit par l'échantillon et à optimiser à l'aide de méthodes non linéaires les paramètres de la simulation, notamment la composition et la structure de la cible, jusqu'à ce que le spectre simulé coïncide avec le spectre expérimental.

\section{Exemples de résultats obtenus par les techniques IBA}

\section{Le couplage des méthodes RBS} et PIXE perce le secret du lustre des céramiques médiévales.

Inventé au $\mathrm{IX}^{\mathrm{e}}$ siècle après J.-C. en Mésopotamie, le lustre est un décor qui orne la céramique glaçurée de prestige (comme la vaisselle) ou de motifs architecturaux. En lumière diffuse, ce décor présente une certaine couleur, mais en réflexion spéculaire sa couleur change et son éclat devient métallique. Ces propriétés optiques sont dues à la présence de nanoparticules de cuivre ou d'argent de 10 à $50 \mathrm{~nm}$ de diamètre, dispersées dans la glaçure. Cette surprenante haute technologie médiévale, qui s'est développée pendant plus de cinq siècles, a pu être étudiée sur des pièces de musée avec des méthodes non destructives.

Si les nanoparticules à l'origine du lustre peuvent être visualisées par microscopie électronique en transmission sur un prélèvement (fig. 2a), le couplage des méthodes PIXE et RBS permet de les étudier directement en respectant l'intégrité de l'objet (photo de la p. 15). Les deux spectres PIXE obtenus avec des protons de $3 \mathrm{MeV}$ donnent la composition globale de la matrice vitreuse et du décor lustré (fig. 2b). En bleu, le spectre acquis sans absorbeur comporte les raies caractéristiques des éléments principaux de la glaçure : c'est un verre plombifere opacifié à l'étain, comportant des traces de cuivre. En rouge, le spectre avec absorbeur permet de détecter la raie de l'argent $\left(\mathrm{Ag}-\mathrm{K}_{\alpha}\right)$ qui correspond aux nanoparticules, sans pouvoir les localiser en profondeur. Le spectre RBS obtenu avec des ions hélium de 2,9 MeV (fig. 2c) permet de tracer le profil de concentration en profondeur des nanoparticules d'argent. En effet, la perte d'énergie subie par les ions incidents lors de la pénétration dans la glaçure et, après rétrodiffusion, le long du trajet vers la sortie, conditionne la forme du spectre RBS : (i) le pic correspondant à la fine couche de nanoparticules d'argent est d'autant plus décalé vers les basses énergies que cette couche est située en profondeur, et sa largeur est liée à l'épaisseur de la couche ; (ii) les éléments distribués de manière homogène dans la glaçure donnent un spectre RBS en forme d'escalier. La position et la forme du pic de l'argent permettent, par un traitement avancé avec le programme SIMNRA, de déterminer la distribution de nanoparticules d'argent au sein de la glaçure. Le profil de concentration obtenu est modélisé par une séquence de couches où l'on observe un gradient de concentration (fig. 2d) [5].

\section{Apport de l'imagerie PIXE-RBS à l'étude des vitraux}

La figure 3 (p. 20) illustre la cartographie combinée PIXE et RBS d'un panneau de vitrail de la cathédrale de Chartres représentant la Vierge à l'Enfant (XIII ${ }^{\mathrm{e}}-\mathrm{XIV}^{\mathrm{e}}$ siècles). La technique de réalisation du décor du vitrail a été consignée au XII ${ }^{\mathrm{e}}$ siècle. Les contours des motifs appliqués sur la plaque de verre, ici les yeux, les sourcils et les mèches de cheveux des personnages, sont réalisés avec une grisaille, mélange à base d'oxydes de cuivre et/ou de fer dans du vin ou de l'urine (fig. 3a). À l'intérieur des contours, les couleurs des carnations et des cheveux sont obtenues en appliquant un lavis. Deux recettes de lavis sont possibles : grisaille diluée ou mélange à base de silicate de plomb, et la question était de savoir laquelle avait été employée. La méthode PIXE a permis de distinguer clairement les deux recettes. La figure $3 b$ représente les répartitions spatiales du calcium (en rouge), du fer (en bleu) et du plomb (en vert), obtenues par PIXE sous faisceau de protons de $3 \mathrm{MeV}$. Le calcium est présent dans le verre substrat du décor. Le fer n'est localisé que dans la grisaille dessinant le décor : l'œil, le sourcil et les mèches de cheveux. Le plomb est quant à lui présent à la fois dans la grisaille et dans le lavis, permettant de conclure que le lavis n'est pas constitué de grisaille diluée mais bien obtenu à partir d'un mélange à base de silicate de plomb. L'intérêt des faisceaux d'ions réside dans la complémentarité des informations 


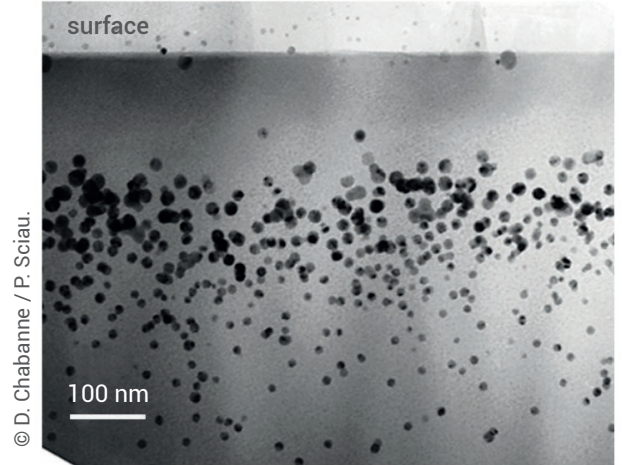

a

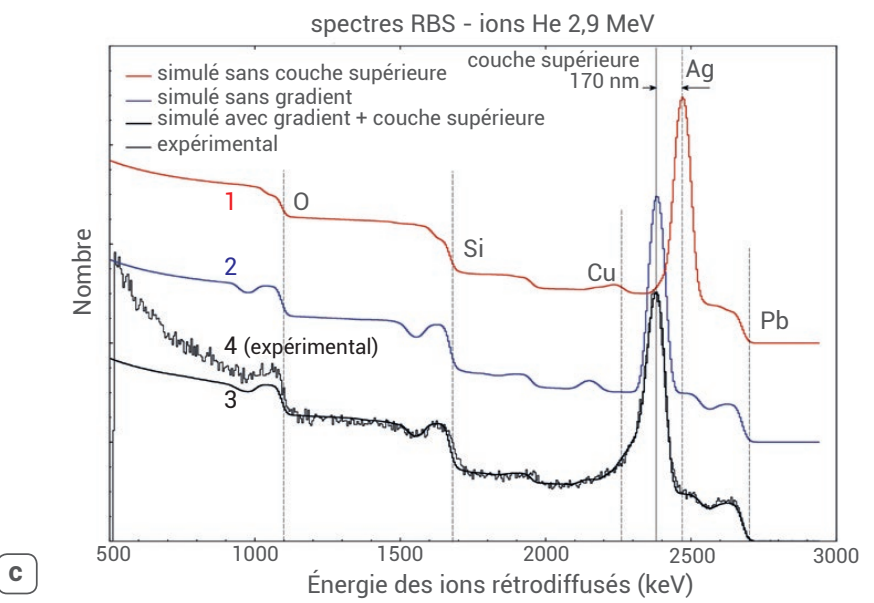

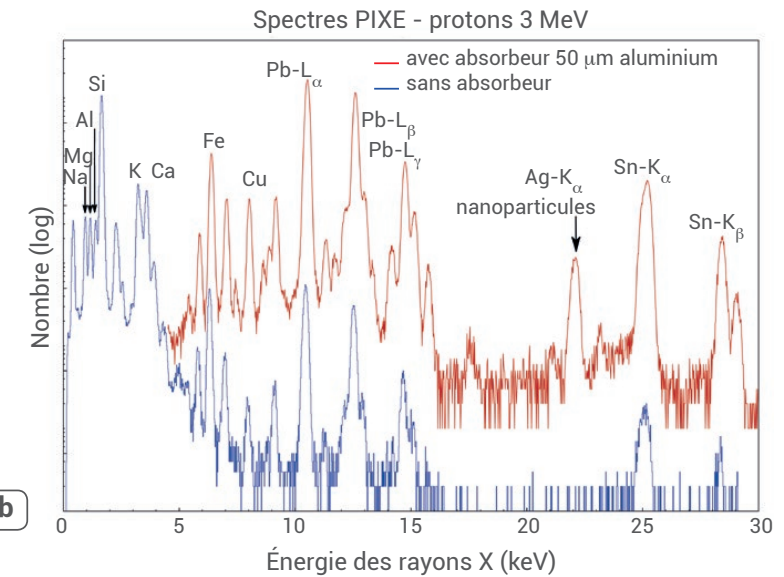

Énergie des rayons $\mathrm{X}(\mathrm{keV})$

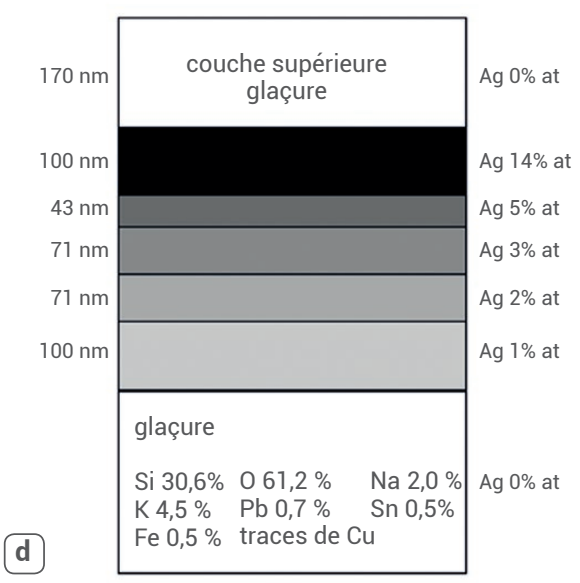

2. Caractérisation du décor en nanoparticules de céramiques médiévales par méthodes RBS et PIXE couplées.

(a) Image de la distribution des nanoparticules du décor lustré, obtenue par microscopie électronique en transmission.

(b) Spectres PIXE obtenus sur la couche du lustre avec des protons de $3 \mathrm{MeV}$. En bleu, le spectre obtenu sans absorbeur comportant les raies caractéristiques des éléments majeurs de la glaçure ( $\mathrm{Si}, \mathrm{Na}, \mathrm{K}, \mathrm{Ca}, \mathrm{Pb}$ et $\mathrm{Sn}$ ). En rouge, spectre obtenu avec une feuille d'aluminium de $50 \mu \mathrm{m}$ d'épaisseur qui absorbe les rayons $X$ caractéristiques de moins de $5 \mathrm{keV}$, permettant la détection d'éléments en faible concentration, notamment l'argent des nanoparticules (raie Ag- $\mathrm{K}_{\alpha}$ ).

(c) Spectre RBS obtenu sur le décor lustré avec des ions hélium de 2,9 MeV. Le spectre présente une forme caractéristique en escalier aux fronts de rétrodiffusion des éléments principaux (indiqués par des lignes verticales en pointillés), à laquelle se superpose un pic dû à la couche de nanoparticules d'argent. Trois étapes du traitement sont représentées : en rouge, le spectre simulé avec un gradient mais sans couche supérieure de glaçure ; en bleu, le spectre simulé avec la couche mais sans gradient; en noir, le spectre simulé avec couche et gradient, qui coïncide avec le spectre expérimental.

(d) Modèle schématique de distribution en profondeur des nanoparticules d'argent, dérivé du traitement du spectre RBS.

recueillies simultanément. La figure $3 c$ représente le spectre de RBS pour l'ensemble de la cartographie, où la bande verticale en bleu correspond au plomb. L'image du dessous (fig. 3d) représente l'intensité de cette bande en fonction de la zone sondée par le faisceau de protons. Il s'agit donc d'une cartographie du plomb dans la partie inférieure de la figure $3 \mathrm{~b}$, mais avec une information supplémentaire que seule la méthode RBS peut fournir : la profondeur à laquelle se trouve le plomb par rapport à la surface. Ces informations complémentaires recèlent de précieux indices sur le pro- cédé de fabrication de ces chefs-d'œuvre. Les outils permettant de visualiser les informations quantitatives tirées de l'exploitation du spectre RBS de chaque pixel sont en cours de développement par l'équipe d'AGLAÉ.

\section{Défis et perspectives}

La place des méthodes IBA en sciences du patrimoine doit être reconsidérée à la lumière de ses atouts et de ses limites.

En effet, bien que ces méthodes soient très présentes dans l'étude du patrimoine, l'émergence d'autres techniques analytiques remet en question leur statut privilégié. D'un côté, l'emploi de méthodes légères et mobiles telles que la spectroscopie Raman, la fluorescence de rayons X portable (p-XRF) ou la spectrométrie d'émission atomique induite par laser (LIBS), se développe rapidement grâce aux progrès de leurs performances et à la possibilité d'analyse in situ dans les musées ou en fouilles. Les méthodes de laboratoire comme la spectrométrie de masse à plasma inductif (ICP-MS) permettent l'analyse d'une gamme très étendue d'éléments avec une excellente sensibilité, au prix d'un microprélèvement. De l'autre, 

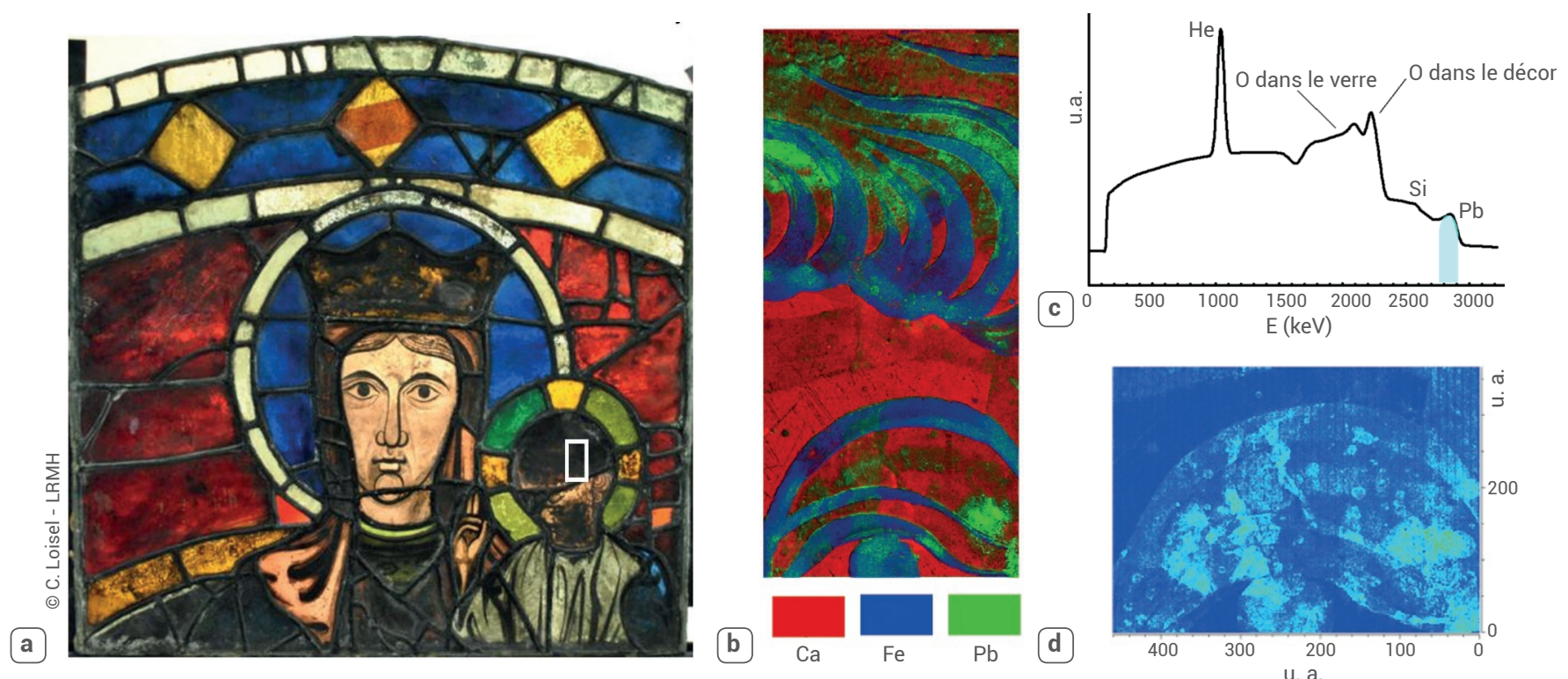

3. Cartographies PIXE et RBS sous faisceau de protons de $3 \mathrm{MeV}$ d'un vitrail de la cathédrale de Chartres (XIII ${ }^{\mathrm{e}}$ siècle).

(a) Le cadre blanc localise la zone cartographiée sur le vitrail.

(b) Cartographie PIXE de cette zone, donnant la répartition des éléments calcium, fer et plomb.

(c) Spectre RBS global du vitrail. En bleu, la zone correspondant à la bande de rétrodiffusion du plomb.

(d) Cartographie RBS de la répartition spatiale du plomb dans la partie inférieure de la figure 3b, correspondant à la bande bleue de la figure 3c.

$\gg>$

l'emploi des grands instruments désormais largement ouverts (synchrotrons, sources de neutrons...) offre des possibilités qui vont bien au-delà de l'analyse élémentaire. Les techniques utilisant le rayonnement synchrotron permettent notamment d'identifier le degré d'oxydation par XANES (spectroscopie d'absorption X près d'un seuil) ou l'environnement chimique par EXAFS (oscillations du coefficient d'absorption des rayons $\mathrm{X}$ ), et la structure cristalline par diffraction X. De même, les faisceaux de neutrons, beaucoup plus pénétrants, permettent de caractériser les matériaux à cœur, notamment par diffraction, ou de déterminer par activation nucléaire des éléments légers, ou difficiles à mesurer comme les terres rares.

L'analyse IBA reste toutefois sans équivalent dans trois domaines :

- la mesure non destructive des éléments légers par PIGE et NRA ;

- le profilage élémentaire en profondeur non destructif par RBS et NRA ;

- l'imagerie élémentaire à grande échelle et à haute résolution spatiale par PIXE ou RBS.

Les méthodes utilisant des faisceaux d'ions constituent aujourd'hui un maillon de la chaine des techniques analytiques au service du patrimoine.

L'analyse IBA fait actuellement face à plusieurs défis, en premier lieu celui de la complexité multi-échelle des matériaux. Les outils de traitement, de simulation et d'interprétation des données acquises sur les objets complexes en sont à leurs balbutiements.

Un autre défi est celui de la sécurité des méthodes IBA. L'exposition à des faisceaux focalisés très intenses ou pendant une longue durée lors de cartographies peut induire des modifications dans les matériaux sensibles, notamment de nature organique. Cela constitue un frein sérieux à l'analyse IBA des objets fragiles comme les peintures de chevalet, en raison de la présence de résines végétales, de liants bio-organiques, de pigments organominéraux, etc.

Des programmes de recherche internationaux rassemblant scientifiques (physiciens, chimistes) et experts du patrimoine (conservateurs, archéologues, historiens d'art, restaurateurs) se sont mis en place pour développer des protocoles d'analyse responsables [6]. L'échange fertile entre les communautés des sciences exactes, notamment de la physique, et du patrimoine a permis de créer et d'adapter des instruments et des méthodes pour mieux comprendre les œuvres d'art et les objets archéologiques. En retour, ces outils innovants et les défis de physique soulevés ont une portée qui dépasse le cadre du patrimoine culturel.

\section{Références}

1 - T. Calligaro et J.-C. Dran, dans Ion Beam Analysis: Fundamentals and Applications, M. Nastasi, J. W. Mayer, Y. Wang eds, Taylor \& Francis, p. 267 (2015).

2- G. Deconninck, "Quantitative analysis by $(p, X)$ and $(p, y)$ reactions at low energies", J. Radioanal. Chem. 12 (1972) 157.

3. L. Giuntini et P.A. Mando, "External beam RBS in an unenclosed helium environment", Nucl. Instr. and Meth. B85 (1994) 744.

4• https://nucleus.iaea.org/sites/ accelerators/knowledgerepository/ code/forms/allitems.aspx

5. D. Chabanne et al., "Physico-chemical analyses of Hispano-Moresque lustred ceramic: a precursor for Italian majolica", Appl. Phys. A, 92 (2008) 11.

\section{L. Bertrand et al., "Mitigation} strategies for radiation damage in the analysis of ancient materials", Trends in Analytical Chemistry 66 (2015) 128.

$7 \cdot$ L. Pichon et al., "Development of a multi-detector and a systematic imaging system on the AGLAE external beam", Nucl. Instr. and Meth. B318 (2014) 27. 